\title{
A Biosurveillance-driven Home Score to Guide Strep Pharyngitis Treatment
}

\author{
Andrew Fine ${ }^{* 1,2}$, Victor Nizet $^{3}$ and Kenneth Mandl ${ }^{1,2}$ \\ 'Boston Children's Hospital, Boston, MA, USA; ${ }^{2 H a r v a r d ~ M e d i c a l ~ S c h o o l, ~ B o s t o n, ~ M A, ~ U S A ; ~}{ }^{3}$ UCSD, La Jolla, CA, USA
}

\section{Objective}

1. To derive and validate an accurate clinical prediction model ("home score") to estimate a patient's risk of group A streptococcal (GAS) pharyngitis before a health care visit based only on history and real-time local biosurveillance, and to compare its accuracy to traditional clinical prediction models composed of history and physical exam features. 2. To examine the impact of a home score on patient and public health outcomes.

\section{Introduction}

GAS pharyngitis affects hundreds of millions of individuals globally each year, and over 12 million seek care in the United States annually for sore throat. Clinicians cannot differentiate GAS from other causes of acute pharyngitis based on the oropharynx exam, so consensus guidelines recommend use of clinical scores to classify GAS risk and guide management of adults with acute pharyngitis. When the clinical score is low, consensus guidelines agree patients should neither be tested nor treated for GAS. A prediction model that could identify very-low risk patients prior to an ambulatory visit could reduce low-yield, unnecessary visits for a most common outpatient condition. We recently showed that real-time biosurveillance can further identify patients at low-risk of GAS. With increasing emphasis on patient-centric health care and the well-documented barriers impeding clinicians' incorporation of prediction models into medical practice, this presents an opportunity to create a patient-centric model for GAS pharyngitis based on history and recent local epidemiology. We refer to this model as the "home score," because it is designed for use prior to a physical exam.

\section{Methods}

Analysis of data collected from 110,208 patients 3 years and older who presented with pharyngitis to a national retail health chain, from 2006-08. Practitioners collected standardized historical and physical exam information based on algorithm-driven care, and all patients with pharyngitis were tested for GAS. We used a previously validated biosurveillance variable reflecting disease incidence called recent local proportion positive (RLPP), which represents the proportion of patients who tested GAS positive in a local market in the previous 14 days. To derive the "home score," candidate variables were restricted to demographic factors, historical items and the RLPP, while physical exam variables (such as exudate), were excluded. Multi- variate analytic techniques were used to identify predictors of GAS. For each home score (0-100), we calculated the percent of patients who tested positive, and we examined the relationship between the home score and GAS positivity. Standard metrics (sensitivity, specificity, positive and negative predictive value, and AUC) were used to compare the performance of the home score to standard scores. We computed the number of patients aged $>=15$ years who, according to the home score, were at low risk for GAS, and therefore might avoid or delay a trip to a medical provider. Outcomes included the numbers of reduced visits and the number of additional missed GAS cases compared to the standard Centor score approach (Do not test/Do not treat if Centor score is 0-1). To facilitate comparison across different risk thresholds, we calculated outcomes for hypothetical cohorts of 1000 patients, and extrapolated these findings to provide the impact on 12 million annual national pharyngitis visits.

\section{Results}

The 3 best predictors were fever (OR 2.43, 95\%CI 2.33-2.54), absence of cough $(1.71,1.63-1.80)$ and RLPP $(1.04,1.04-1.04$ per unit change in RLPP). Using a home score cutoff of 0.10 to identify adults at low risk would save 230,000 ambulatory visits annually while missing only 8500 additional GAS cases. At a 0.20 cutoff, 2.9 million visits would be saved, and 320,000 more cases missed each year. There was a strong correlation between the percent testing positive and the home score ( $\mathrm{r}$-square $=0.98)$. As the home score increases, there is a linear increase in the risk of GAS (slope=1.02). The home score AUC was 0.66, approaching the Centor score (0.69) even without any physical exam information.

\section{Conclusions}

A biosurveillance-driven home score to guide treatment of strep pharyngitis could save millions of visits annually by identifying patients in the pre-visit setting who would be unlikely to be tested or treated.

\section{Keywords}

biosurveillance; pharyngitis; retail health

*Andrew Fine

E-mail: andrew.fine@childrens.harvard.edu 